\title{
Scanning transmission X-ray microscopy studies of chromium hydroxide hollow spheres and nanoparticles formed by gamma-radiation
}

\begin{tabular}{|c|c|}
\hline Journal: & Canadian Journal of Chemistry \\
\hline Manuscript ID & cjc-2017-0142.R1 \\
\hline Manuscript Type: & Article \\
\hline Date Submitted by the Author: & 18-Apr-2017 \\
\hline Complete List of Authors: & $\begin{array}{l}\text { Wang, Zhiqiang; Western University, Chemistry } \\
\text { Alrehaily, Leena; Western University, Chemistry } \\
\text { Joseph, Jiju; Western University, Chemistry } \\
\text { Wren, Jungsook; The University of Western Ontario } \\
\text { Wang, Jian; Canadian Light Source Inc } \\
\text { Sham, Tsun-Kong; Department of Chemistry, }\end{array}$ \\
\hline $\begin{array}{l}\text { Is the invited manuscript for } \\
\text { consideration in a Special } \\
\text { Issue?: }\end{array}$ & TK Sham \\
\hline Keyword: & $\begin{array}{l}\mathrm{Cr}(\mathrm{OH}) 3 \text {, Scanning transmission } \mathrm{X} \text {-ray microscopy, } \mathrm{X} \text {-ray absorption near } \\
\text { edge structures, hollow spheres, gamma radiolysis }\end{array}$ \\
\hline
\end{tabular}


1 Scanning transmission X-ray microscopy studies of chromium hydroxide hollow spheres

2 and nanoparticles formed by gamma-radiation

3 Zhiqiang Wang, ${ }^{1}$ Leena Alrehaily, ${ }^{1}$ Jiju Joseph, ${ }^{1}$ Jungsook Clara Wren, ${ }^{*},{ }^{1}$ Jian Wang ${ }^{2}$ and Tsun-

$4 \quad$ Kong Sham *,1

$5{ }^{1}$ Department of Chemistry, the University of Western Ontario, London, Ontario N6A 5B7,

6 Canada

$7 \quad{ }^{2}$ Canadian Light Source Inc., University of Saskatchewan, Saskatoon, Canada S7N 0X4

8 * Author to whom correspondence should be addressed. Email: tsham@uwo.ca and

$9 \quad$ jcwren@uwo.ca

10

11

12

13

14

15

16

17

18

19 
20 Abstract: Amorphous chromium-containing solid nanoparticles and hollow spheres were

21 formed as the intermediate product during the synthesis of $\mathrm{Cr}_{2} \mathrm{O}_{3}$ by gamma radiolysis of

22 dichromate solution. Scanning transmission X-ray microscopy (STXM) is applied to investigate

23 the electronic structure and chemical composition of this intermediate product (solid

24 nanoparticles and hollow spheres). STXM result obtained at O K-edge and $\mathrm{Cr}_{3,2}$-edgeconfirms

25 that chromium in the sample exists as $\mathrm{Cr}^{3+}$, and the solid nanoparticles and hollow spheres are

26 both $\mathrm{Cr}(\mathrm{OH})_{3}$. Combined with other techniques, a mechanism for gamma-radiation-induced

27 formation of $\mathrm{Cr}_{2} \mathrm{O}_{3}$ nanoparticles via $\mathrm{Cr}(\mathrm{OH})_{3}$ intermediatesfrom dissolved dichromate is

28 proposed.

29 Keywords

30 Chromium hydroxide, hollow spheres, gamma radiolysis, Scanning transmission X-ray

31 microscopy, X-ray absorption near edge structure

\section{Introduction}

33 Chromium (III) oxide, one of the principal oxides of chromium, is an antiferromagnetic insulator

34 below theNéel temperature $307 \mathrm{~K} .{ }^{1} \mathrm{Cr}_{2} \mathrm{O}_{3}$ adopts the corundum structure, consisting of a

35 hexagonal close packed array of oxide anions with $2 / 3$ of the octahedral holes occupied by

36 chromium. $\mathrm{Cr}_{2} \mathrm{O}_{3}$ nanoparticles have been used in a broad range of applications, such as green

37 pigments, ${ }^{2}$ heterogeneous catalysts, ${ }^{3}$ coating materials for thermal protection, ${ }^{4}$ coatings for wear

38 resistance, ${ }^{5}$ and electrochromic materials. ${ }^{6}$ Different applications require different crystalline

39 particle size and morphology. $\mathrm{Cr}_{2} \mathrm{O}_{3}$ nanoparticles have been synthesized by various techniques

40 including hydrothermal reduction, ${ }^{7}$ solution combustion synthesis, ${ }^{8}$ sonochemical reaction, ${ }^{9}$

41 laser-induced pyrolysis, ${ }^{10}$ hydrazine reduction and thermal treatments, ${ }^{11}$ supercritical alcohol 
42 synthesis, ${ }^{12}$ condensation-polymerization, ${ }^{13}$ precipitation-gelation, ${ }^{14}$ gas condensation, ${ }^{15}$

43 microwave plasma chemistry, ${ }^{16}$ and sol-gel methods. ${ }^{17}$ The major drawbacks of most of these

44 fabrication methods are a wide particle-size distribution, a low yield, and agglomeration of the

45 particles. The fabrication methods also tend to be complex and require chemically harsh

46 conditions and/or high processing temperatures.

47 Radiolysis is a promising new technique for generating nanoparticles with a narrow size distribution. Radiation-induced nanoparticle formation also does not require the chemical

49 additives and stabilizers for particle size control that are used in other conventional methods. It

50 eliminates undesired effects of these chemicals on the structure and chemical composition of

51 nanoparticles. This method has been successfully used to fabricate $\gamma$-FeOOH, ${ }^{18} \mathrm{Co}_{3} \mathrm{O}_{4}{ }^{19}$ and

$52 \mathrm{Cr}_{2} \mathrm{O}_{3}$ nanoparticles. ${ }^{20-21}$ However the mechanistic studies of the nanoparticle formation process

53 via radiolysis are very limited. ${ }^{18,20}$ In the case of $\mathrm{Cr}_{2} \mathrm{O}_{3}$ nanoparticles, the

54 precipitations(intermediate product)obtained from $\mathrm{Cr}_{2} \mathrm{O}_{7}{ }^{2-}$ solution show different morphologies

55 and sizes (solid nanoparticles with different diameters and hollow spheres) and very poor

56 crystallinity. ${ }^{20-21}$ Therefore it is hard to determine the chemical composition of this intermediate

57 product (the solid particles and hollow spheres) via conventional techniques such as X-ray

58 diffraction (XRD), laboratory X-ray photoelectron spectroscopy (XPS) and transmission electron

59 microscopy (TEM). This information is important for the understanding ofthe mechanism and

60 therefore to better control the morphology and size of thefinal product $\mathrm{Cr}_{2} \mathrm{O}_{3}$.

61 Using the brilliant, undulator-based and polarization-controlled third-generation synchrotron

62 sources, scanning transmission X-ray microscopy (STXM) provides an excellent combination of

63 chemical speciation (X-ray absorption near edge structures, XANES) and microscopy with a 
64 spectral resolution and a spatial resolution of $0.05 \mathrm{eV}$ and of $30 \mathrm{~nm}$, respectively. STXM

65 employs a nano-sized X-ray beam and provides spectral-microscopic information by measuring

66 the absolute absorption of the specimen in transmission mode; thus both chemical images and

67 detailed absorption spectroscopic features of a single nanostructure (including its thickness) can

68 be revealed. STXM has been successfully applied to study an individual nanostructure and

69 microstructure with different phases and compositions. ${ }^{22-25}$ Herebyusing STXM, we reported the

70 electronic structures of chromium hydroxide hollow spheres and solid nanoparticles that

71 wereformed as the intermediate product during the synthesis of $\mathrm{Cr}_{2} \mathrm{O}_{3}$ via gamma-irradiation.

72 STXM result at O K-edge confirms that the hollow spheres and the solid nanoparticles with

73 different diameters are both $\mathrm{Cr}(\mathrm{OH})_{3}$. After a dehydration process, amorphous $\mathrm{Cr}(\mathrm{OH})_{3}$ hollow

74 spheres and solid nanoparticles were transformed to crystalline $\mathrm{Cr}_{2} \mathrm{O}_{3}$ nanoparticles.

\section{Experimental}

76 The chromium sample was prepared by gamma-irradiation of dichromate solution $(0.1 \mathrm{mM})$

77 containing $0.1 \mathrm{M}$ tert-butyl alcohol (purity $\geq 99 \%$, Sigma-Aldrich) under deaerated condition

78 (Argon). Chromium solution was prepared by dissolving high-purity potassium dichromate $(\geq$

$7999 \%$, Sigma-Aldrich) in water purified using a NANO pure Diamond UV ultrapure water system

80 (resistivity of $18.2 \mathrm{M} \Omega \cdot \mathrm{cm}$ ). The $\mathrm{pH}$ of the test solution was adjusted to $6.0 \mathrm{using} \mathrm{NaOH}$ solution.

81 Using a syringe, $10 \mathrm{~mL}$ of the chromium solution was transferred to a pre-sealed $20-\mathrm{mL}$ vial

82 (Agilent Technologies) purged with argon gas. To the capped vial the appropriate volume of tert-

83 butyl alcohol was injected to get the desired concentration $(0.1 \mathrm{M})$. The test vial was irradiated in

$84 \mathrm{a}^{60} \mathrm{Co} \gamma$ cell (MDS Nordion) for $300 \mathrm{~min}$ as described in a previous paper. ${ }^{20}$ The $\gamma$ radiation

85 source provided a uniform absorption dose rate of $4 \mathrm{kGy} \cdot \mathrm{h}^{-1}$ in the water samples at the time of 
this study. After that the test solution was extracted with a syringe, and then was centrifuged and washed with distilled water a few times.

The morphology of the as-prepared sample was first characterized by TEM (Philips CM10). STXM measurement was carried out at the SM beamline of the Canadian Light Source (CLS), which is equipped with a $25 \mathrm{~nm}$ outermost-zone zone plate (CXRO, Berkeley Lab). The diffraction-limited spatial resolution for this zone plate is $30 \mathrm{~nm}$. We measured the same copper grid that was previously used for TEM. Image sequence (stack) scans over a range of photon energies were acquired for the same sample region at the $\mathrm{Cr}_{3,2}$-edge and $\mathrm{O} \mathrm{K}$-edge. STXM data were analyzed using the aXis2000 software package, which allows for detailed interactive processing of the images and fitting of the X-ray absorption spectra contained in the image stacks. More details of STXM measurement and data analysis can be found elsewhere. ${ }^{23,25}$

The $\mathrm{Cr} \mathrm{L}_{3,2}$-edge and $\mathrm{O}$ K-edge XANES of pure $\mathrm{Cr}(\mathrm{OH})_{3}$ and $\mathrm{Cr}_{2} \mathrm{O}_{3}$ powders were collected at the SGM beamline of CLS using total electron yield (TEY) mode.All TEY-XANES were normalized with respect to the intensity of the incoming beam $\left(I_{0}\right)$.

\section{Results and discussions}

The morphology of the as-prepared sample is first characterized by TEM. As shown in Figure 1, with irradiation time of $300 \mathrm{~min}$, the sample is composed of large hollow spheres with diameter of 100-170 nm and wall thickness of about $14-18 \mathrm{~nm}$ and small solid nanoparticles (thesmallersolid nanoparticles with light contrast have a diameter of 8-20 nm, while those bigger ones with dark contrast have a diameter of 40-50 $\mathrm{nm}$ ). Previously it is proposed that the large hollow spheres were $\mathrm{Cr}(\mathrm{OH})_{3}$ and the small solid nanoparticles were $\mathrm{Cr}_{2} \mathrm{O}_{3}{ }^{20}$

XANES is an element- and site-specific technique, which is sensitive to the local chemical environment of a specific element (no matter the sample is amorphous or crystalline). STXM is a 
109

110

111

112

113

114

115

116

117

118

119

120

121

122

123

124

125

126

127

128

129

130

131

spectromicroscopy technique that allows for the acquisition of XANES spectrum from a specific region of interest (ROI) on an individual nanostructure. By comparing the XANES collected from the hollow spheres and solid nanoparticles, it will help us determine their composition.Figure 2a is a STXM optical density image of thesample that shows thesamearea as in the TEM image (see Figure 1). One can see clearly the hollow spherical structures. Because the spatial resolution of the $25 \mathrm{~nm}$ zone plate is $30 \mathrm{~nm}$, the morphology of the small nanoparticles is not well resolved.

Since STXM can determine the absolute thickness of the sample based on X-ray absorption, the thickness distribution of the hollow spheres and solid nanoparticles has been obtained and is shown in Figure 3. The thickness was obtained from stack fitting with the quantitatively scaled reference spectra of $1 \mathrm{~nm}$ thickness at O K-edge (Figure S1 in Supprting Information). The wall thickness of the hollow spheres is determined to be about $15-20 \mathrm{~nm}$, which agrees well with the TEM observation.The thickness of the solid particles in the red, blue and green dashed circles are 5-10 $\mathrm{nm}$ (nanoparticles with small diameter), 25-40 $\mathrm{nm}$ (nanoparticles with medium diameter) and 60-75 $\mathrm{nm}$ (nanoparticles with large diameter), respectively. The brightest area marked with purple dotted circle shows thickness of $120-138 \mathrm{~nm}$, which is due to the aggregation of solid particles (the darkest area in Figure 1).

Now let us compare the XANES collected from the large hollow spheres and small solid particles to determine their composition.Figure 4 shows $\mathrm{Cr} \mathrm{L}_{3,2}$-edge XANES spectrum isolated from the colored regions of interest (ROIs) in Figure 2b, which probes the electron transitions from $\mathrm{Cr} 2 p$ to the previously unoccupied $\mathrm{Cr} 3 d$ and $4 s$ states. $^{26}$ The $2 p$ core hole spin-orbit interaction splits the absorption spectrum by approximately $8 \mathrm{eV}$ into an $\mathrm{L}_{3}$-edge $\left(2 p_{3 / 2}\right)$ and $\mathrm{L}_{2^{-}}$ edge $\left(2 p_{1 / 2}\right)$.Let us first look at the spectrum of pure $\mathrm{Cr}(\mathrm{OH})_{3}$ and $\mathrm{Cr}_{2} \mathrm{O}_{3}$. The XANES of 
$132 \mathrm{Cr}(\mathrm{OH})_{3}$ shows almost the same line shape as that of $\mathrm{Cr}_{2} \mathrm{O}_{3}$. The only difference is that the 133 feature $B$ in the spectrum of $\mathrm{Cr}_{2} \mathrm{O}_{3}$ locates at slightly lower energy than that of $\mathrm{Cr}(\mathrm{OH})_{3}(\Delta E=$ $1340.25 \mathrm{eV}$ ). The XANES collected from hollow spheres (ROI-1, ROI-2, ROI-3, ROI-4, ROI-5 and 135 ROI-6 marked in Figure 2b) show the same features as that from solid particles (ROI-7, ROI-8 136 and ROI-9 marked in Figure 2b).The feature $B$ in these nano-XANES is closer to that in $\mathrm{Cr}(\mathrm{OH})_{3}$ 137 than $\mathrm{Cr}_{2} \mathrm{O}_{3}$.At $\mathrm{Cr} \mathrm{L}_{3,2}$-edge, due to the subtle difference between the XANES of $\mathrm{Cr}(\mathrm{OH})_{3}$ and 138 $\mathrm{Cr}_{2} \mathrm{O}_{3}$, it is hard to determine the composition of hollow spheres and solid nanoparticles. But it can be concluded that the $\mathrm{Cr}$ in the sample only exists as $\mathrm{Cr}^{3+}$, because that $\mathrm{Cr}^{6+}$ (from the precursor $\mathrm{Cr}_{2} \mathrm{O}_{7}{ }^{2-}$ ), $\mathrm{Cr}^{4+}$ (possible reduction product) and $\mathrm{Cr}^{2+}$ (possible reduction product) have 141 very different XANES features. ${ }^{26-27}$

142 Let us move to the O K-edge. Figure 5 displays O K-edge nano-XANES isolated from the same 143 ROIslabeled in Figure $2 \mathrm{~b}$ as well as XANES of pure $\mathrm{Cr}(\mathrm{OH})_{3}$ and $\mathrm{Cr}_{2} \mathrm{O}_{3}$. These spectra probes 144 the transitionfrom $\mathrm{O} 1 s$ to unoccupied states with at least partial $\mathrm{O} 2 p$ character hybridized with $\mathrm{Cr}$ $1453 d$ states. ${ }^{28}$ Taking the $\mathrm{O} \mathrm{K}$-edge XANES of $\mathrm{Cr}_{2} \mathrm{O}_{3}$ for example, the spectrum is divided into two 146 regions. The first region shows a sharp peak $a$ ' near energy threshold $E_{0}$ followed by a weak 147 shoulder $b^{\prime}$. The sharp peak $a^{\prime}$ can be assigned to a superposition of the $t_{2 g \downarrow}$ state and the $e_{g \uparrow}$ state 148 with a total number of five electrons. ${ }^{28}$ The shoulder $b$ ' relates to the remaining two $e_{g} \downarrow$ 149 electrons. ${ }^{28}$ The second region, typically $5-15 \mathrm{eV}$ above $E_{0}$, is attributed to $\mathrm{O} p$ character 150 hybridized with $\mathrm{Cr} 4 s$ and $4 p$ states. $^{28}$ In the spectrum of $\mathrm{Cr}(\mathrm{OH})_{3}$, feature $a$ and $b$ locate at 151 higher energy compared to feature $a^{\prime}$ and $b^{\prime}$ of $\mathrm{Cr}_{2} \mathrm{O}_{3}$. The energy difference between peak $a$ of $152 \mathrm{Cr}(\mathrm{OH})_{3}$ and peak $a^{\prime}$ of $\mathrm{Cr}_{2} \mathrm{O}_{3}$ is about $0.8 \mathrm{eV}$. Moreover, the peak $a$ of $\mathrm{Cr}(\mathrm{OH})_{3}$ is much less 153 intense than the peak $a^{\prime}$ of $\mathrm{Cr}_{2} \mathrm{O}_{3}$. The presence of the $\mathrm{OH}$ bonding significantly reduces the 154 unoccupied states of $p$ character at the O K-edge whiteline. With such spectroscopic differences 
155

156

157 158

159

160

161

162

163

164

165

166

167

168

169

170

171

172

173 174 does not exist in the sample $\left(\mathrm{Cr}^{4+}\right.$ has a sharp peak just below $\left.530 \mathrm{eV}^{29}\right)$, which is consistent with 175 Cr L ${ }_{3,2}$-edge XANES results (see Figure 4).

176 Combined STXM result with TEM, FTIR and XPS results, ${ }^{20}$ a mechanism for gamma-radiation-

177 induced formation of $\mathrm{Cr}_{2} \mathrm{O}_{3}$ nanoparticles from dissolved dichromate is proposed. The radiation-

between these two compound, it provides an opportunity to identify the composition of the hollow spheres and solid nanoparticles.

As shown in Figure 5, O K-edge XANES collected from hollow spheres (ROI-1, ROI-2, ROI-3, ROI-4, ROI-5 and ROI-6 marked in Figure 2b) shows similar features as that from solid particles (ROI-7, ROI-8 and ROI-9 marked in Figure 2b). The peak $a$ and $b$ in these spectra locate at the same energy as that of $\mathrm{Cr}(\mathrm{OH})_{3}$.Furthermore, the intensity ratio between peak $a$ and $b$ in these spectra is much closer to that of $\mathrm{Cr}(\mathrm{OH})_{3}$ than $\mathrm{Cr}_{2} \mathrm{O}_{3}$. All the above evidence indicate that both the hollow spheres and the solid nanoparticles with different diameters are of dominantly $\mathrm{Cr}(\mathrm{OH})_{3}$ character. One can see that feature $a$ is broadened and slightly weaker than feature $b$ in the spectrum of some hollow spheres (such as ROI-1, ROI-2 and ROI-3)compared to that of standard $\mathrm{Cr}(\mathrm{OH})_{3}$. Since the sample thickness is about $30-40 \mathrm{~nm}$ (the wall thickness of hollow spheres is estimated to be 15-20 nm based on the thickness distribution shown in Fig. 3), self absorption effect that usually leads to the peak broadening in the XANES spectrum (especially for soft X-ray) will not account for this broadening. Here the broadening of feature $a$ is proposed to be due to thepresence of some $\mathrm{Cr}_{2} \mathrm{O}_{3}$ (feature $a^{\prime}$ in the spectrum of $\mathrm{Cr}_{2} \mathrm{O}_{3}$ locates at lower energy).The " $b$ " feature at ROI 1-3 appears to be more intense than that expected in the $\mathrm{Cr}(\mathrm{OH})_{3}$ standard. This may be due to texture effect resulting from the higher strain of the spheres. The " $b$ " feature becomes more $\mathrm{Cr}(\mathrm{OH})_{3}$ like in ROI 4-6 where the spheres get larger and in ROI 7-9 where solid particles areexamined (Figure 2).The O K-edge XANES also confirms that $\mathrm{Cr}^{4+}$ 
178 induced formation of $\mathrm{Cr}_{2} \mathrm{O}_{3}$ nanoparticles occurs in four stages. Stage I involves radiolytic

179 reduction of $\mathrm{Cr}^{\mathrm{VI}}(\mathrm{aq})$ to $\mathrm{Cr}^{\mathrm{III}}(\mathrm{aq})$ species in the solution phase, followed by spontaneous

180 condensation of $\mathrm{Cr}(\mathrm{OH})_{3}$ particles. This provides sites onto which $\mathrm{Cr}^{\mathrm{VI}}$ and $\mathrm{Cr}^{\mathrm{III}}$ coprecipitate and

181 form mixed $\mathrm{Cr}^{\mathrm{VI}} / \mathrm{Cr}^{\mathrm{III}}$ oxide/hydroxide particles. In stage II, radiolytic interconversion of $\mathrm{Cr}^{\mathrm{VI}}(\mathrm{aq})$

182 to $\mathrm{Cr}^{\mathrm{III}}(\mathrm{aq})$ in solution reaches a steady state, while the adsorption of $\mathrm{Cr}^{\mathrm{VI}}$ on particles continues,

183 growing mixed $\mathrm{Cr}^{\mathrm{VI}} / \mathrm{Cr}^{\mathrm{III}}$ oxide/hydroxide particles. In stage III, solid-state conversion of $\mathrm{Cr}^{\mathrm{VI}}$

184 and $\mathrm{Cr}^{\mathrm{III}}$ in the particles slowly converts the oxide from a mixed $\mathrm{Cr}^{\mathrm{VI}} / \mathrm{Cr}^{\mathrm{III}}$ oxide/hydroxide to

185 mainly $\mathrm{Cr}(\mathrm{OH})_{3}$. At this stage, the sample (as collected/unwashed)consists of large amount of

186 nanoparticles with different sizes. The small particles are $\mathrm{Cr}(\mathrm{OH})_{3}$ with a diameter ranging10-50

$187 \mathrm{~nm}$. While the large oneswith size of 140-200 $\mathrm{nm}$ is proposed to be of core/shell structure.

188 Chromium hydroxide is the shell and some $\mathrm{Cr}^{\mathrm{VI}}$ species that was not yet converted to $\mathrm{Cr}(\mathrm{OH})_{3}$ is

189 the core. Therefore, once the sample was washed with distilled water, TEM and STXM image

190 show small solid nanoparticles and large hollow spheres that are both $\mathrm{Cr}(\mathrm{OH})_{3}$. At the last stage,

$191 \mathrm{Cr}(\mathrm{OH})_{3}$ solid nanoparticles and hollow spheres were converted to $\mathrm{Cr}_{2} \mathrm{O}_{3}$ nanoparticles after

192 dehydration process (heated at $400{ }^{\circ} \mathrm{C}$ for $5 \mathrm{~h}$ in air). It should be noted that it has been reported

193 that hexagonal hollow $\mathrm{Cr}(\mathrm{OH})_{3}$ pellets have been observed, which upon heating converts to

$194 \mathrm{Cr}_{2} \mathrm{O}_{3} \cdot{ }^{30}$

\section{Conclusion}

196 The electronic structures of chromium hollow spheres and solid nanoparticles prepared from

197 dissolved dichromate via gamma-radiation have been obtained by STXM at $\mathrm{Cr}_{3,2}$-edge and O

198 K-edge.Although whether or not the hollow spheres and solid particles are $\mathrm{Cr}(\mathrm{OH})_{3}$ or $\mathrm{Cr}_{2} \mathrm{O}_{3}$ can

199 not be conclusively identified at the $\mathrm{Cr} \mathrm{L}_{3,2}$-edge, it is confirmed that $\mathrm{Cr}$ only exists as $\mathrm{Cr}^{3+}$.

200 However,the O K-edge nano-XANES collected from big hollow spheres (single sphere) and 
201 small solid nanoparticles (particle assembly) shows solid evidence that both the hollow spheres 202 and the solid particles are $\mathrm{Cr}(\mathrm{OH})_{3}$, which is consistent with the XPS results (Here STXM with

203 transmission mode gives both surface and bulk information of the sample, while laboratory XPS 204 only surface information).Thus STXM results help better understand the structure and bonding 205 of the products, hence the mechanism of radiation-induced formation of $\mathrm{Cr}_{2} \mathrm{O}_{3}$ nanoparticles 206 from dissolved dichromate.

207 Acknowledgements

208 We would like to thank beamline scientists Tom Regier and James Dynes for their technical 209 support at CLS. Research at the University of Western Ontario is supported by NSERC, CFI, 210 OIT, OMRI and CRC (TKS). CLS is supported by CFI, NSERC, CHIR, NRC, and the 211 University of Saskatchewan.

\section{References}

213 1. Punugupati, S.; Narayan, J.; Hunte, F., J Appl Phys 2015,117 (19). doi: Artn 193907

$21410.1063 / 1.4921435$.

215 2. Brock, T.; Groteklaes, M.; Mischke, P. European Coating Handbook; Vincentz:

216 Hannover, Germany, 2000.

217 3. Abu-Zied, B. M., Appl Catal a-Gen 2000,198 (1-2), 139. doi: Doi 10.1016/S0926-

$218 \quad 860 x(99) 00508-6$.

219 4. Berdahl, P., J Heat Trans-T Asme 1995,117 (2), 355. doi: Doi 10.1115/1.2822529.

220 5. Kitsunai, H.; Hokkirigawa, K.; Tsumaki, N.; Kato, K., Wear 1991,151 (2), 279. doi: Doi

$221 \quad 10.1016 / 0043-1648(91) 90255-S$.

222 6. T Ivanova, K. G., A Cziraki, A Szekeres and E Vlaikova Journal of Physics: Conference 223 Series 2008,113, 012030. doi: 10.1088/1742-6596/113/1/012030. 
224 7. Pei, Z. Z.; Xu, H. B.; Zhang, Y., J Alloy Compd 2009,468 (1-2), L5. doi:

$225 \quad 10.1016 /$ j.jallcom.2007.12.086.

$226 \quad 8 . \quad$ Lima, M. D.; Bonadimann, R.; de Andrade, M. J.; Toniolo, J. C.; Bergmann, C. P., J Eur 227 Ceram Soc 2006,26 (7), 1213. doi: 10.1016/j.jeurceramsoc.2005.01.042.

228 9. Dhas, N. A.; Koltypin, Y.; Gedanken, A., Chem Mater 1997,9 (12), 3159. doi: Doi $229 \quad 10.1021 / \mathrm{Cm} 9704645$.

230 10. Peters, G.; Jerg, K.; Schramm, B., Mater Chem Phys 1998,55 (3), 197. doi: Doi

231 10.1016/S0254-0584(98)00121-7.

232 11. Gui, Z.; Fan, R.; Mo, W. Q.; Chen, X. H.; Yang, L.; Hu, Y., Mater Res Bull 2003,38 (1), 233 169. doi: Pii S0025-5408(02)00983-2

234 Doi 10.1016/S0025-5408(02)00983-2.

235 12. Znaidi, L.; Pommier, C., Eur J Sol State Inor 1998,35 (6-7), 405. doi: Doi

236 10.1016/S0992-4361(98)80019-2.

237 13. Kawabata, A.; Yoshinaka, M.; Hirota, K.; Yamaguchi, O., J Am Ceram Soc 1995,78 (8), 238 2271. doi: Doi 10.1111/J.1151-2916.1995.Tb08655.X.

239 14. Kim, D. W.; Shin, S. I.; Lee, J. D.; Oh, S. G., Mater Lett 2004,58 (12-13), 1894. doi: $240 \quad$ 10.1016/j.matlet.2003.11.023.

241 15. Balachandran, U.; Siegel, R. W.; Liao, Y. X.; Askew, T. R., Nanostruct Mater 1995,5 (5), 242 505. doi: Doi 10.1016/0965-9773(95)00266-H.

243 16. Vollath, D.; Szabo, D. V.; Willis, J. O., Mater Lett 1996,29 (4-6), 271. doi: Doi

$244 \quad 10.1016 / \mathrm{S} 0167-577 \times(96) 00158-9$.

245 17. Zhu, C. C.; Hong, Y.; Zhang, J. G.; Zhou, B. B., Key Eng Mat 2007,353-358, 2111. 
246 18. Yakabuskie, P. A.; Joseph, J. M.; Keech, P.; Botton, G. A.; Guzonas, D.; Wren, J. C., 247 Phys Chem Chem Phys 2011,13 (15), 7198. doi: 10.1039/c1cp20084d.

248 19. Alrehaily, L. M.; Joseph, J. M.; Biesinger, M. C.; Guzonas, D. A.; Wren, J. C., Phys 249 Chem Chem Phys 2013,15 (3), 1014. doi: 10.1039/c2cp43094k.

250 20. Alrehaily, L. M.; Joseph, J. M.; Wren, J. C., J Phys Chem C 2015,119 (28), 16321. doi: $251 \quad$ 10.1021/acs.jpcc.5b02540.

252 21. Alrehaily, L. M.; Joseph, J. M.; Musa, A. Y.; Guzonas, D. A.; Wren, J. C., Phys Chem 253 Chem Phys 2013,15 (1), 98. doi: 10.1039/c2cp43150e.

254 22. Li, J.; Wang, Z. Q.; Zhao, A. K.; Wang, J.; Song, Y.; Sham, T. K., J Phys Chem C 255 2015,119(31), 17848. doi: 10.1021/acs.jpcc.5b04276.

256 23. Guo, X. X.; Wang, Z. Q.; Wu, J.; Wang, J.; Zhu, Y. J.; Sham, T. K., Nanoscale 2015,7 257 (15), 6767. doi: 10.1039/c4nr07471h.

258 24. Wang, Z. Q.; Wang, W.; Sham, T. K.; Yang, S. G., Nanoscale 2014,6 (16), 9783. doi: 259 10.1039/c4nr02231a.

260 25. Wang, Z. Q.; Wang, J.; Sham, T. K.; Yang, S. G., J Phys Chem C 2012,116 (18), 10375. 261 doi: 10.1021/jp301289x.

262 26. Theil, C.; van Elp, J.; Folkmann, F., Phys Rev B 1999,59 (12), 7931. doi: Doi $26310.1103 /$ Physrevb.59.7931.

264 27. Zhang, K. H. L.; Du, Y.; Sushko, P. V.; Bowden, M. E.; Shutthanandan, V.; Sallis, S.; 265 Piper, L. F. J.; Chambers, S. A., Phys Rev B 2015,91 (15). doi: Artn 155129

$266 \quad$ 10.1103/Physrevb.91.155129.

267 28. Degroot, F. M. F.; Grioni, M.; Fuggle, J. C.; Ghijsen, J.; Sawatzky, G. A.; Petersen, H., 268 Phys Rev B 1989,40 (8), 5715. doi: Doi 10.1103/Physrevb.40.5715. 
269 29. Tsujioka, T.; Mizokawa, T.; Okamoto, J.; Fujimori, A.; Nohara, M.; Takagi, H.; Yamaura, 270 K.; Takano, M., Phys Rev B 1997,56 (24), R15509. doi: Doi 10.1103/Physrevb.56.R15509.

271 30. Bai, Y. K.; Zheng, R. T.; Gu, Q.; Wang, J. J.; Wang, B. S.; Cheng, G. A.; Chen, G., J

272 Mater Chem A 2014,2 (32), 12770. doi: 10.1039/c4ta00999a.

273

274

275

276

277

278

279

280

281

282

283

284

285

286

287

288

289

290

291 


\section{Figure Captions}

293 Figure 1 TEM image of the chromium nanoparticlesformed by gamma radiolysis of dichromate 294 solution. The scale bar is $200 \mathrm{~nm}$.

295

296 Figure 2 (a) STXM optical density image of the chromium nanoparticles (the image was

297 averaged from all stack images at the $\mathrm{O}$ K-edge and $\mathrm{Cr} \mathrm{L}_{3,2}$-edge). (b) STXM optical density 298 image at $\mathrm{Cr} \mathrm{L}_{3,2}$-edge $(\mathrm{E}=578 \mathrm{eV})$. The colored masks were created on different regions of 299 interest (ROIs, e.g.: big hollow spheres and small solid nanoparticles).

300

301

Figure 3 Thickness distribution of the chromium hollow spheres and nanoparticles obtained at $\mathrm{O}$

302 K-edge. The vertical bar tracks the thickness.

303

304 Figure $4 \mathrm{Cr} \mathrm{L}_{3,2}$-edge XANES spectra extracted from the ROIs shown in Fig. 2b. Cr L 3,2 -edge 305 XANES of standard $\mathrm{Cr}_{2} \mathrm{O}_{3}$ and $\mathrm{Cr}(\mathrm{OH})_{3}$ is also shown for comparison. The spectra are up shifted 306 on the y axis for clarity.

307

308

Figure 5 O K-edge XANES spectra extracted from the ROIs shown in Fig. 2b. O K-edge XANES of standard $\mathrm{Cr}_{2} \mathrm{O}_{3}$ and $\mathrm{Cr}(\mathrm{OH})_{3}$ is also shown for comparison. The spectra are up shifted on the y axis for clarity. 


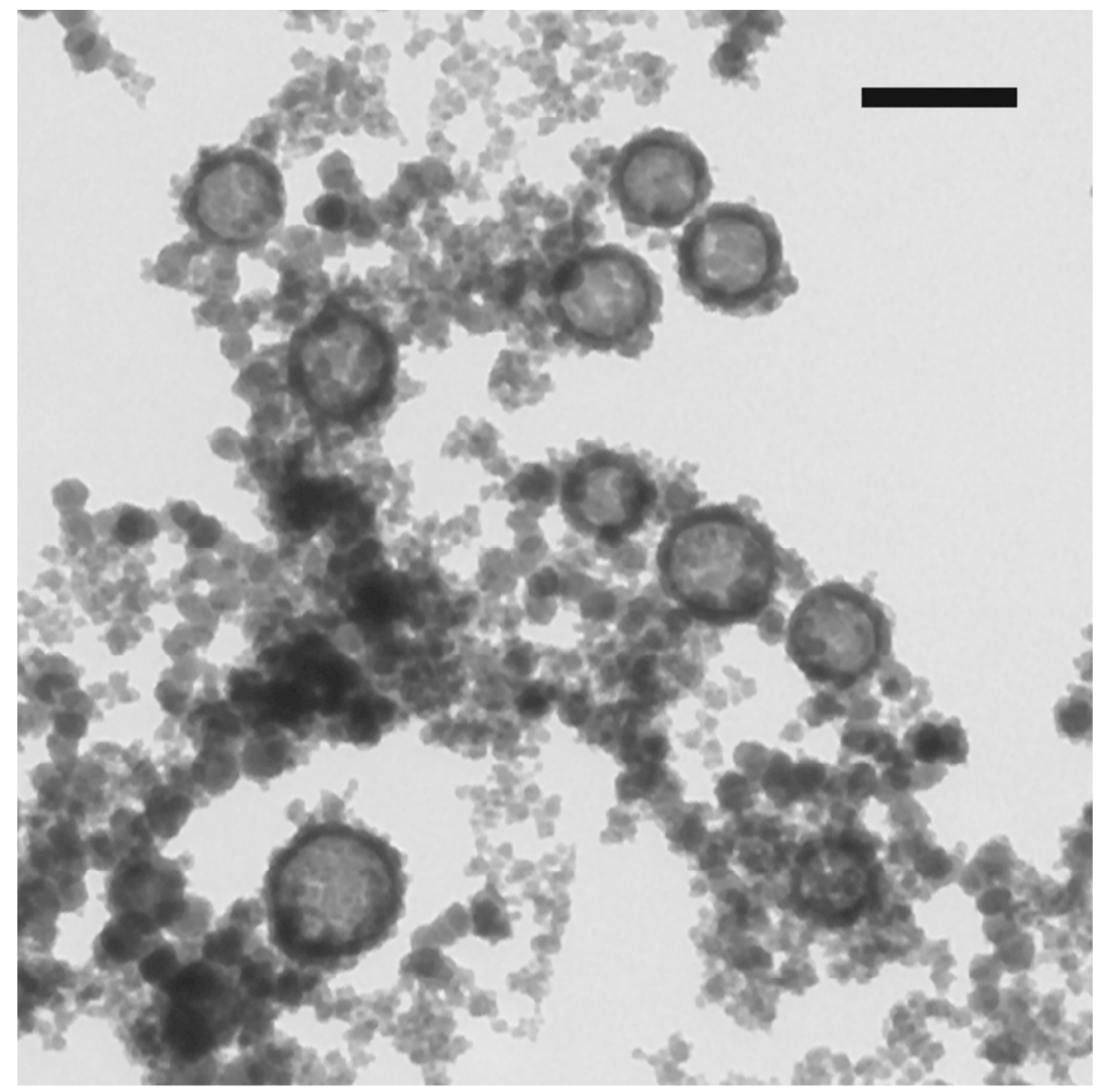

Figure 1 TEM image of the chromium nanoparticles formed by gamma radiolysis of dichromate solution. The scale bar is $200 \mathrm{~nm}$.

$85 \times 85 \mathrm{~mm}(300 \times 300 \mathrm{DPI})$ 


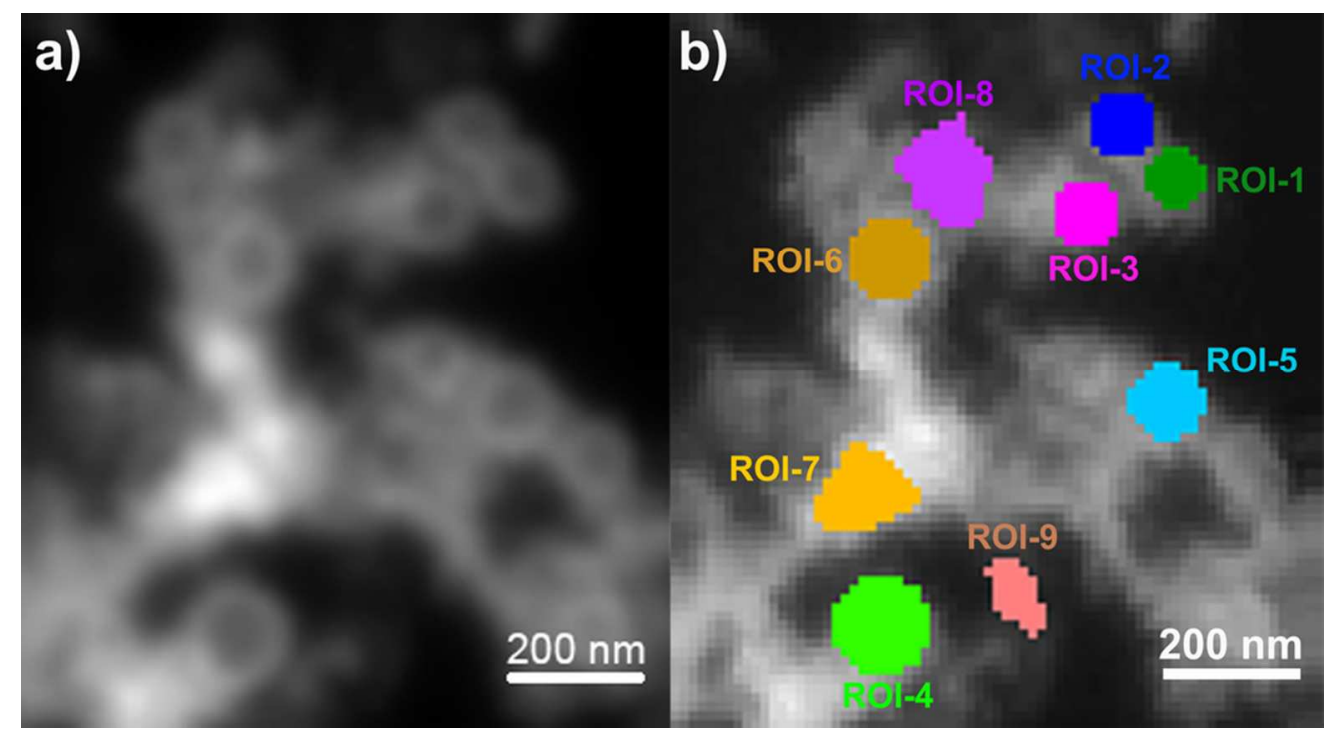

Figure 2 (a) STXM optical density image of the chromium nanoparticles (the image was averaged from all stack images at the $\mathrm{O}$ K-edge and Cr L3,2-edge). (b) STXM optical density image at Cr L3,2-edge (E = 578 $\mathrm{eV}$ ). The colored masks were created on different regions of interest (ROIs, e.g.: big hollow spheres and small solid nanoparticles).

$87 \times 48 \mathrm{~mm}(300 \times 300$ DPI $)$ 


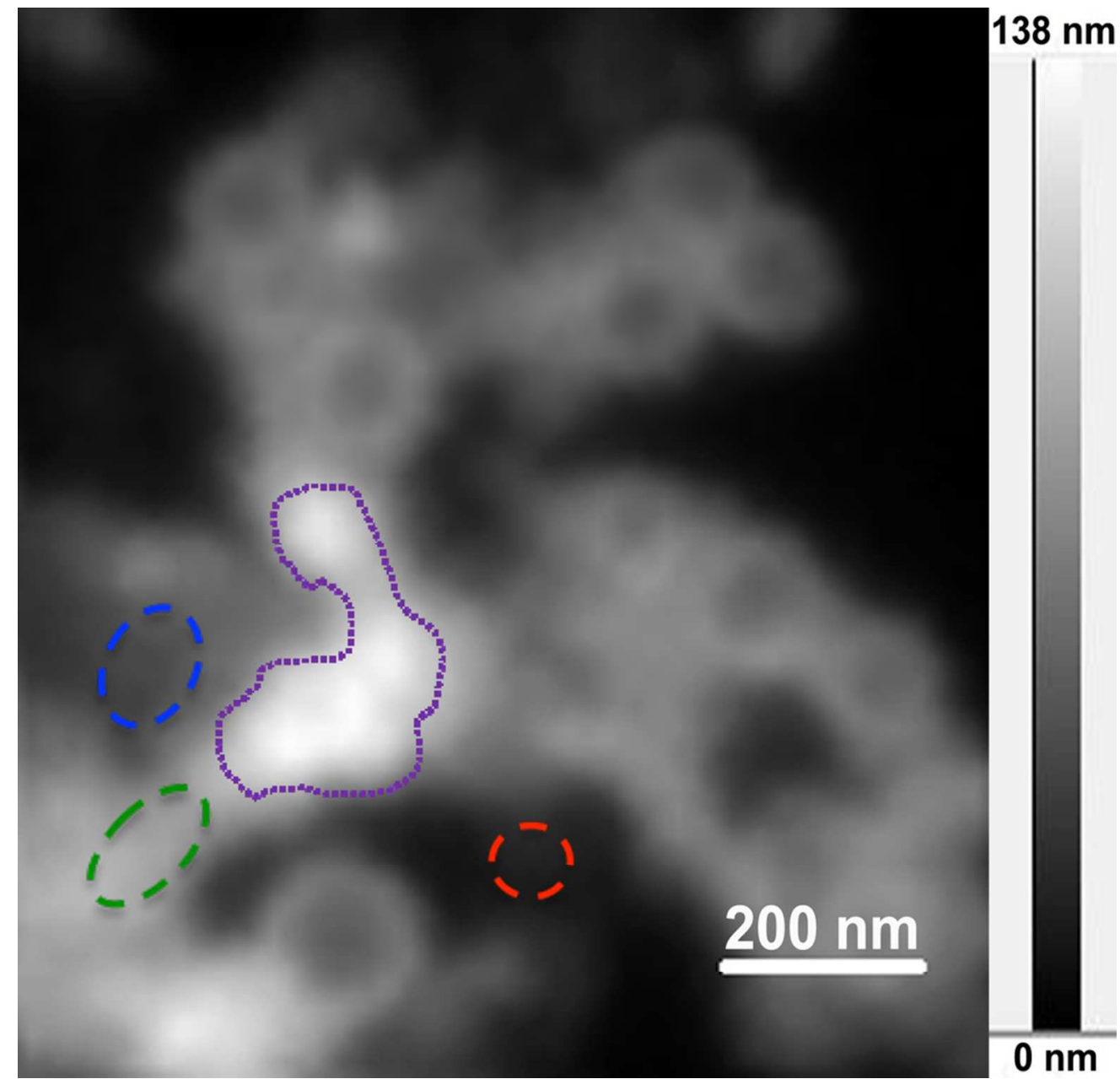

Figure 3 Thickness distribution of the chromium hollow spheres and nanoparticles obtained at O K-edge. The vertical bar tracks the thickness.

$82 \times 80 \mathrm{~mm}(300 \times 300$ DPI $)$ 


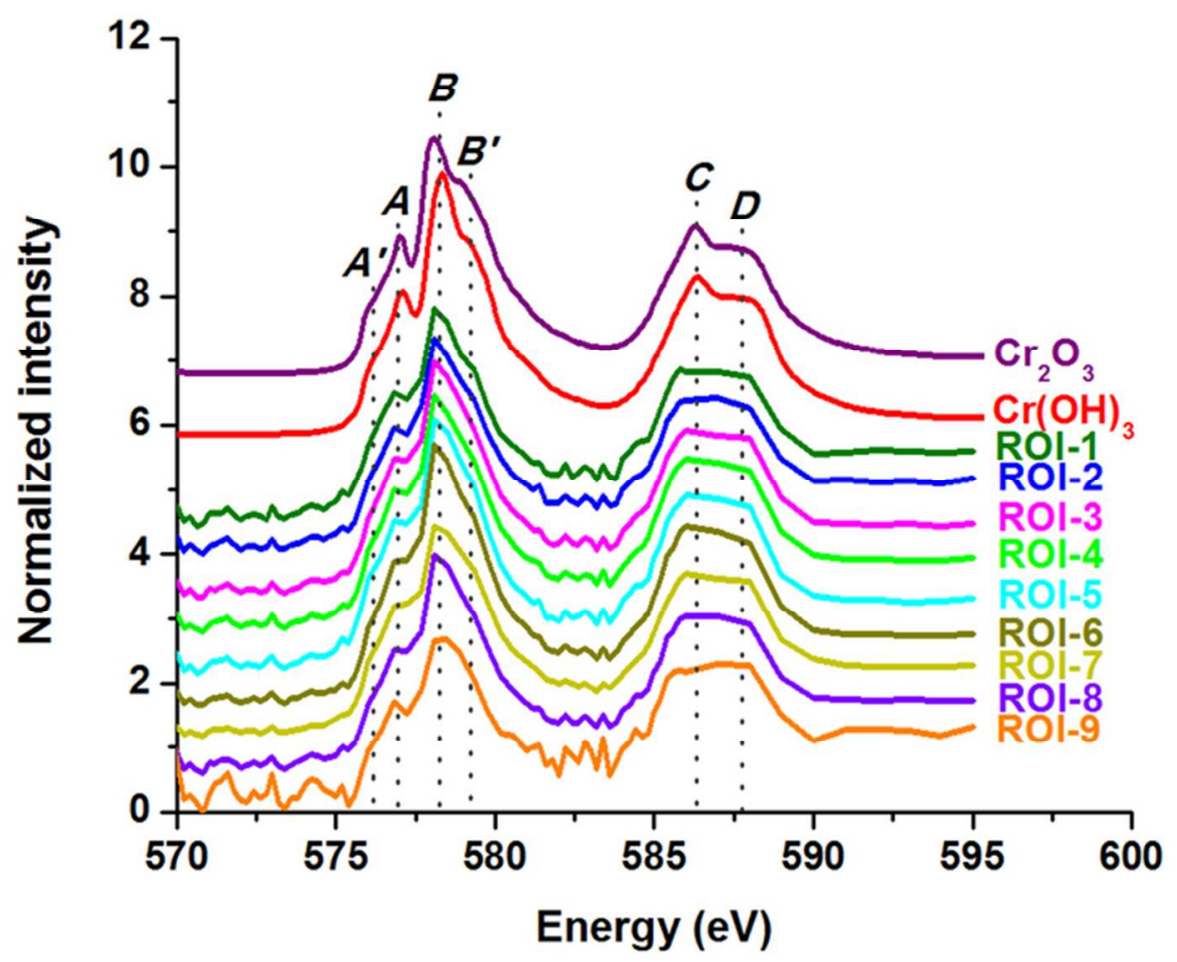

Figure $4 \mathrm{Cr}$ L3,2-edge XANES spectra extracted from the ROIs shown in Fig. 2b. Cr L3,2-edge XANES of standard $\mathrm{Cr} 2 \mathrm{O} 3$ and $\mathrm{Cr}(\mathrm{OH}) 3$ is also shown for comparison. The spectra are up shifted on the $y$ axis for clarity.

$67 \times 54 \mathrm{~mm}(300 \times 300 \mathrm{DPI})$ 


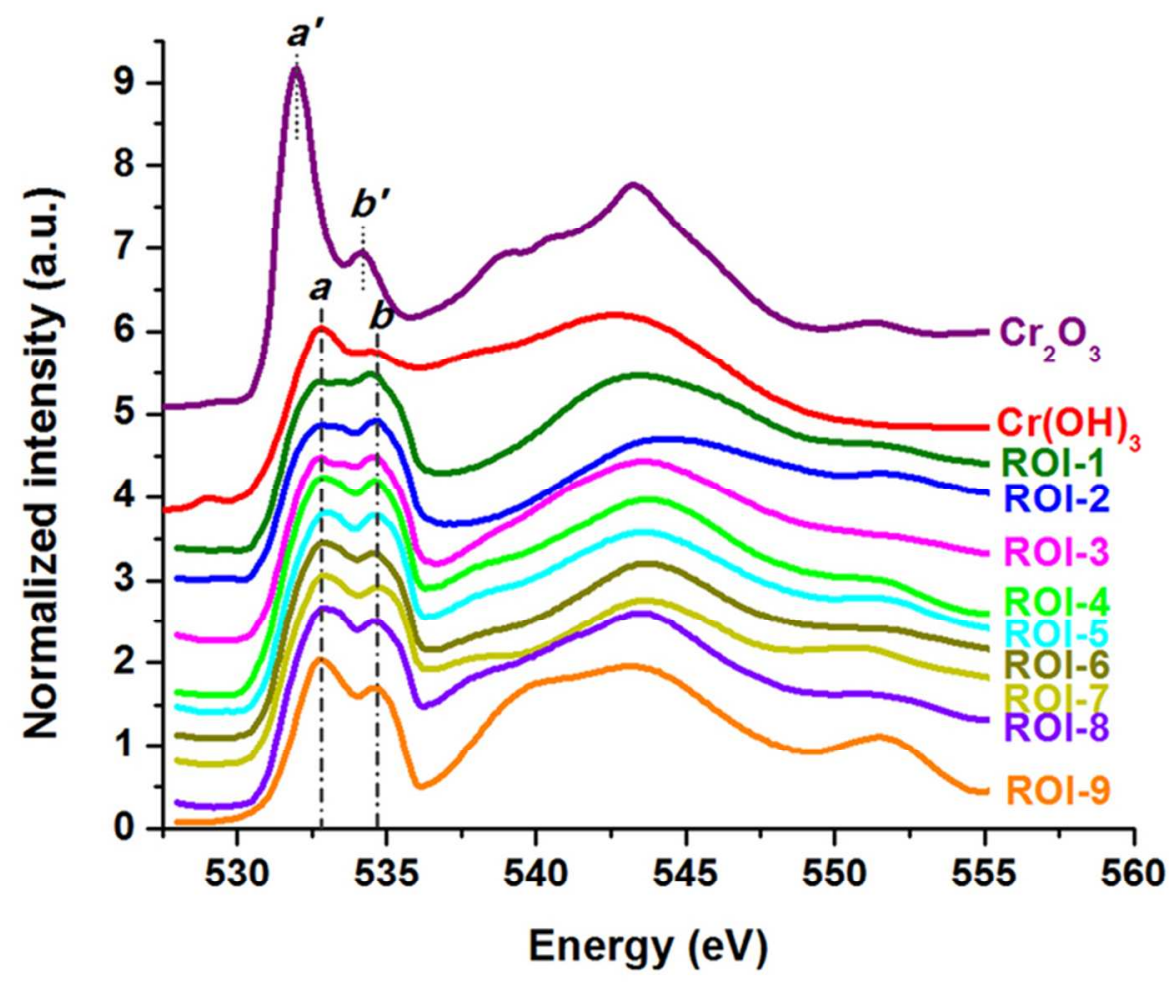

Figure $5 \mathrm{O}$ K-edge XANES spectra extracted from the ROIs shown in Fig. 2b. O K-edge XANES of standard $\mathrm{Cr} 2 \mathrm{O} 3$ and $\mathrm{Cr}(\mathrm{OH}) 3$ is also shown for comparison. The spectra are up shifted on the $y$ axis for clarity.

$67 \times 54 \mathrm{~mm}(300 \times 300 \mathrm{DPI})$ 